THE aim of the current study was to investigate the effect of inhibition of nitric oxide (NO) production after injury on inflammatory cell accumulation and fibrosis around digital flexor tendon and synovium. A standard crush injury was applied to the flexor tendons of the middle digit of the hindpaw and the overlying muscle and synovium of female Wistar rats. Thirty animals received an intraperitoneal injection of either isotonic saline or $N(G)$-nitro-1-arginine methyl ester (L-NAME; $5 \mathrm{mg} / \mathrm{kg}$ ) immediately following the crush injury, and five animals were then sacrificed at various intervals and the paws processed for histology. Another group of five animals was sacrificed after 3 days for nitrite determinations. The results showed that nitrite production and hence NO synthase activity is doubled at the acute phase of tendon wound healing, and we can prevent this by administering a single dose of L-NAME immediately after injury. The incidence and severity of fibrocellular adhesions between tendon and synovium was much more marked in animals treated with L-NAME. Treatment with L-NAME elicited a chronic inflammatory response characterised by a persistent and extraordinarily severe accumulation of large numbers of inflammatory cells in the subcutaneous tissues, in muscle and in tendon. These findings indicate that in the case of injured tendon and synovium, NO could act to protect the healing tissue from an uncontrolled inflammatory response.

Key words: Tendon, Nitric oxide, Crush injury, Adhesion, Fibrosis

\section{Single dose of inducible nitric oxide synthase inhibitor induces prolonged inflammatory cell accumulation and fibrosis around injured tendon and synovium}

\author{
Homa Darmani ${ }^{1, C A}$, James C. Crossan ${ }^{2}$ and \\ Adam Curtis ${ }^{3}$
}

${ }^{1}$ Department of Applied Biology, Faculty of Science, Jordan University of Science and Technology, P.O. Box 3030, Irbid 22110, Jordan; ${ }^{2}$ Department of Orthopaedic Surgery, Western Infirmary, Glasgow, UK; ${ }^{3}$ Centre for Cell Engineering, Department of Infection and Immunity, Glasgow University, Glasgow, UK
${ }^{\mathrm{CA}}$ Corresponding author:
Tel: +962795978834
Fax: +962 27278962
E-mail: darmani@just.edu.jo

\section{Introduction}

Despite numerous advances in surgical techniques, formation of adhesions still remains a major problem in the repair of tendon injuries. Immediately after flexor tendon injury and surgical repair, intrinsic healing by tenocytes and extrinsic healing by inflammatory cells from the tendon sheath are both thought to occur. ${ }^{1}$ Therefore, extrinsic adhesions formed between the tendon and the tendon sheath are part of the normal healing process. ${ }^{1}$ Following injury, post-trauma fibrous adhesions bind adjacent synovial tissue to injured tendon, resulting in adhesion formation and subsequent pain, stiffness and disability.

In an attempt to stop peritendinous adhesion formation, administration of antibodies to transforming growth factor-beta (TGF- $\beta$ ), in the early stages of wound healing, has proved to be of great benefit. ${ }^{1,2}$ It has been established that TGF- $\beta 1$ is upregulated after tendon injury and repair in the tendon-wound environment. ${ }^{3}$ Furthermore, the cytokine TGF- $\beta$ plays an important role in wound healing and fibrosis, ${ }^{2}$ and during wound healing the ability to downregulate such an effector molecule may be as important as its upregulation in order to avoid excess fibrosis. Manipulation of the TGF- $\beta 1$, TGF- $\beta 2$, and TGF- $\beta 3$ isoforms by inhibiting their expression, activation, or signalling has been reported to greatly reduce scarring and fibrosis in animal models. ${ }^{4,5}$ Interestingly, one of the multitude biological actions of TGF- $\beta$ is the inhibition of nitric oxide (NO) production by macrophages through decreasing the stability and translation of mRNA for the enzyme nitric oxide synthase (NOS), as well as increasing the enzyme degradation. ${ }^{6,7}$

Within the past two decades $\mathrm{NO}$, a highly reactive free radical produced from the amino acid arginine by the enzyme NOS, has gained much attention as a potent multifunctional signal molecule with widespread action within and between diverse tissues. ${ }^{8,9}$ It has recently been reported that NO regulates wound healing and that inhibition of NO synthesis may be directly related to impaired healing of wounds. ${ }^{10}$ Indeed, fibroblasts from inducible nitric oxide synthase (iNOS) knockout mice are reported to proliferate more slowly, synthesise less collagen, and contract fibroblast-populated collagen lattices more slowly than wild-type fibroblasts. ${ }^{10}$ Furthermore, 
collagen synthesis was restored to normal in fibroblasts taken from iNOS knockout mice in response to NO donors (s-nitroso- $N$-acetylpenicillamine). Thus, iNOS deficiency causes significant impairment in wound healing-related properties of fibroblasts, which confirms that NO plays an important role in wound healing. ${ }^{10,11}$ On the other hand, it has also been reported that NO may be an important antifibrotic molecule. ${ }^{12}$ Long-term treatment of rats with $N(\mathrm{G})$-nitro-l-arginine methyl ester (L-NAME), an isoform-non-specific inhibitor of NOS, leads to fibrosis of the heart suggesting that NO may play a role in preventing tissue fibrosis. ${ }^{12}$ Ferrini et al. ${ }^{12}$ reported that iNOS induction occurs in fibrotic plaque of both the human and rat Peyronie's disease and that the NO derived from iNOS appears to counteract reactive oxygen species (ROS) formation and collagen deposition. Because the inhibition of iNOS activity leads to a decrease in the NO/ROS ratio, thereby favouring the development of fibrosis, it was proposed that iNOS induction in this tissue may be a protective mechanism against fibrosis and abnormal wound healing. ${ }^{12}$

Recently, it has been reported that all three NOS isoforms are expressed during tendon healing, with differential expression patterns during the various phases of tendon healing. ${ }^{13}$ In the current study we hypothesised that NO may play a beneficial role in the healing of tendon injuries and so we examined the effects of suppression of NO production on inflammatory cell accumulation and fibrosis around injured tendon and synovium. We administered an inhibitor to NOS, L-NAME, in the early stages of wound healing, immediately after inflicting a standard open crush injury of rat digital flexor tendon and synovium.

\section{Materials and methods}

\section{Reagents}

Tissue culture reagents were purchased from Gibco BRL (Life Technologies, Paisley, UK). All other reagents were purchased from Sigma Chemical Co. (Poole, Dorset, UK), unless otherwise stated.

\section{Animal model}

Female Wistar rats weighing in the range of 250-300 $\mathrm{g}$ were anaesthetised using Halothane. Under tourniquet control, a longitudinal incision was made in the plantar surface of the hindpaw and extended along the line of the middle digit to expose the synovial canal. A standard crush injury was applied with Adson's forceps along the length of the flexor tendons of the middle digit of the hindpaw and the overlying muscle and synovium. The wound was sutured using a continuous nylon suture. The animals were allowed to mobilise without restriction following the procedure. The animals were then sacrificed by $\mathrm{CO}_{2}$ asphyxiation followed by cervical dislocation at various time points following injury.

\section{Control uninjured group}

Ten animals were sacrificed, five for histology and five for nitrite determinations.

\section{Crush injury group}

Thirty animals received an intraperitoneal injection of isotonic saline immediately following crush injury. Five animals were then sacrificed after 3 days, 2, 3, 4 and 5 weeks, and the paws were then processed for histology. Another group of five animals was sacrificed after 3 days for nitrite determinations.

\section{Crush injury group treated with L-NAME}

Thirty animals received an intraperitoneal injection of L-NAME $(5 \mathrm{mg} / \mathrm{kg})$ immediately following the crush injury. Five animals were then sacrificed after 3 days, 2, 3, 4 and 5 weeks, and the paws were then processed for histology. Another group of five animals was sacrificed after 3 days and 5 weeks for nitrite determinations.

\section{Preparation of specimens for histology}

Tissue was prepared for histological examination by undertaking a block dissection to include the wounded skin, subcutaneous tissues, tendon, synovium and muscle, all of which were carefully dissected from the underlying bones. The dissected tissues were pinned onto cork to achieve good alignment of the tendons and were then fixed in $10 \%$ neutral buffered formalin and processed for paraffin embedding. Paraffin sections were prepared in $5 \mu \mathrm{m}$ sections and stained with haematoxylin and eosin dyes by standard procedures.

\section{Tissue immunohistochemistry}

Block dissected specimens, treated as already described, were embedded in OCT medium (Miles Inc., Elkhart, IN, USA) and frozen to $-70^{\circ} \mathrm{C}$. Longitudinal sections, $5 \mu \mathrm{m}$ in thickness, were cut onto slides, allowed to reach room temperature, and then processed for immunohistochemical staining of cells expressing the macrophage marker ED1. 


\section{Immunohistochemical staining for macrophages using ED1}

The sections were washed in Tris-buffered saline (TBS), and treated with dilute normal serum for 15 min. The slides were then drained and incubated with mouse anti-rat macrophage clone ED1 (Serotec, Oxford, UK) (prepared in TBS and bovine serum albumin) for $1 \mathrm{~h}$ at room temperature. After washing three times in TBS for $5 \mathrm{~min}$, the slides were treated with biotin-conjugated secondary antibody for 45 min at room temperature. Subsequent to three further 5 min washes in TBS, the sections were incubated with alkaline phosphate-conjugated extravidin (Sigma) at a dilution of 1:1000 for $30 \mathrm{~min}$. The sections were washed three times in TBS and then visualised using fast 5-bromo-4-chloro-3 indolyl phosphate/nitro-blue tetrazolium substrate (Sigma).

\section{Preparation of specimens for nitrite determinations}

Dissected tissue (wounded skin, subcutaneous tissues, tendon, synovium and muscle) was carefully separated from the underlying bones. The tissue was minced into small pieces and gently disrupted using a pestle and mortar. Organ cultures were then rinsed in saline and maintained in RPMI supplemented with $10 \%$ foetal calf serum, $100 \mu \mathrm{g} / \mathrm{ml}$ of penicillin, $100 \mathrm{U} /$ $\mathrm{ml}$ of streptomycin, $2.85 \mathrm{mM}$ glutamine and bicarbonate for a total of 8 days at $37^{\circ} \mathrm{C}$. The release of $\mathrm{NO}$ was measured as nitrite, a stable byproduct of NO.

\section{Nitrite assay}

Fifty microlitres of the culture supernates or tissue extracts were mixed with an equal volume of Griess reagent, which consisted of $1 \%$ sulfanilamide, $0.1 \%$ $N$ (1-naphthyl)-ethylene diamine dihydrochloride and $2.5 \%$ phosphoric acid, and left in the dark for $20 \mathrm{~min}$ at room temperature. A BioRad multiscan microtitre plate reader was used for the determination of the absorbance at $540 \mathrm{~nm}$, which is proportional to the concentration of nitrite in the medium.

\section{Results}

Figure 1 shows that in digital flexor tendon/synovium explants from uninjured rats, the explants released nitrite under basal conditions and thus showed iNOS activity. The crush injured group that did not receive L-NAME were sacrificed at the acute phase of the inflammatory response (3 days) and explant cultures from these animals showed a statistically significant increase in levels of nitrite release, when compared with explants from control uninjured

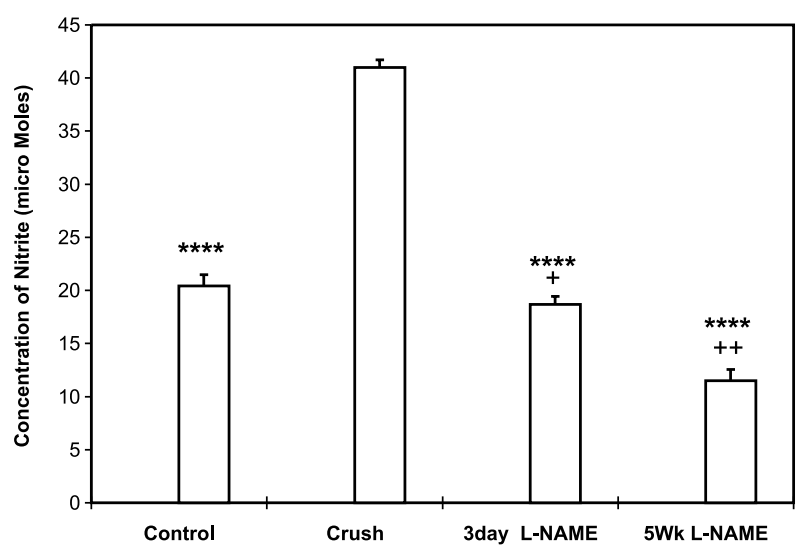

FIG. 1. Release of nitrite from explant cultures of digital flexor tendon and synovium. Explant cultures were taken from (i) control uninjured rats, (ii) crush injured rats after 3 days, and rats treated with L-NAME immediately after crush injury and sacrificed (iii) 3 days and (iv) 5 weeks post-injury. Explant cultures were incubated for 3 days at $37^{\circ} \mathrm{C}$ and the accumulation of nitrite, the stable endproduct of NO, was measured in the supernatant, as described previously. Error bars represent standard error of the means. ${ }^{* * *} p<0.0001$ versus crush injury (no L-NAME); ${ }^{+} p<0.05$ and ${ }^{+}{ }^{+} p<0.005$ versus control.

animals $(p<0.0001)$. There is greater than a $100 \%$ increase in NOS activity in crush injured paws.

When L-NAME was administered immediately after the crush injury and the animals sacrificed at the acute phase of the inflammatory response, namely 3 days, a marked and statistically significant reduction in levels of nitrite was observed $(p<0.0001$ versus crush injury without L-NAME) (Fig. 1).

When L-NAME was administered immediately after the crush injury and the animals sacrificed 5 weeks after the injury, the explant cultures still showed a statistically significant reduction in the levels of nitrite release in comparison with the control uninjured group $(p<0.005$ versus control uninjured group). Five weeks after a crush injury, the inflammatory response in crush injured paws would settle almost completely and we would expect the NOS activity in the crush injured paws to be slightly higher or very close to the control uninjured group.

Figure 2 shows the histological appearance of normal uninjured paws, and it can be seen that tendon and synovium are separated by a clear synovial canal. Epitenon cells on the tendon surface and the synovial lining cells are one layer thick and there are few inflammatory cells in the subsynovial tissues.

Figures 3-6 show the histological appearance of the normal wound healing process and the wound healing process in rats that had been administered a single dose of the iNOS inhibitor L-NAME immediately following injury, at various times (namely, 3 days and 2, 3, 4 and 5 weeks subsequent to wounding). 


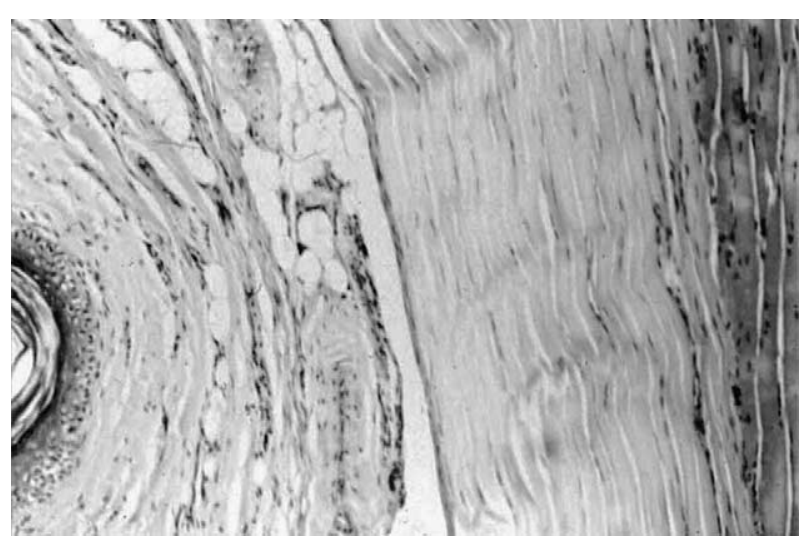

FIG. 2. Uninjured flexor tendon, synovial sheath and subcutaneous tissue in the rat ( $\times 10$, haematoxylin and eosin). Tendon (right) and synovium (left) are separated by a clear synovial canal. The normal synovial and subsynovial tissues contain modest numbers of fibroblasts and inflammatory cells.

\section{Three days}

An intense inflammatory cell infiltration was present in the subcutaneous and subsynovial tissues in control injuries (Fig. 3A). The subcutaneous tissues were edematous and somewhat disorganised, and were heavily infiltrated by inflammatory cells that were mainly of monocyte/macrophage lineage. In addition, scattered mast cells were present in the subsynovial tissues and often lay in close proximity to the injured tendon. In some areas the inflammatory cells accumulated in clusters containing numerous cells. In addition, moderate numbers of leukocytes were present in the adventitial spaces between injured muscle bundles.

In animals treated with a single intraperitoneal injection of L-NAME, the injured tissues were characterised by edema in the subcutaneous tissues, which were heavily infiltrated by a population of mononuclear cells that were larger and that had larger nuclei than those seen in control injured paws (Fig. 3B). Mast cells were also present in larger numbers than in control injured paws. The characteristic feature that distinguished the paws of animals treated with L-NAME was the extraordinarily severe adherence of large numbers of mononuclear cells to tendon and to muscle (Fig. 3C,D, respectively). These features were not apparent in any of the control injured paws.
(A)

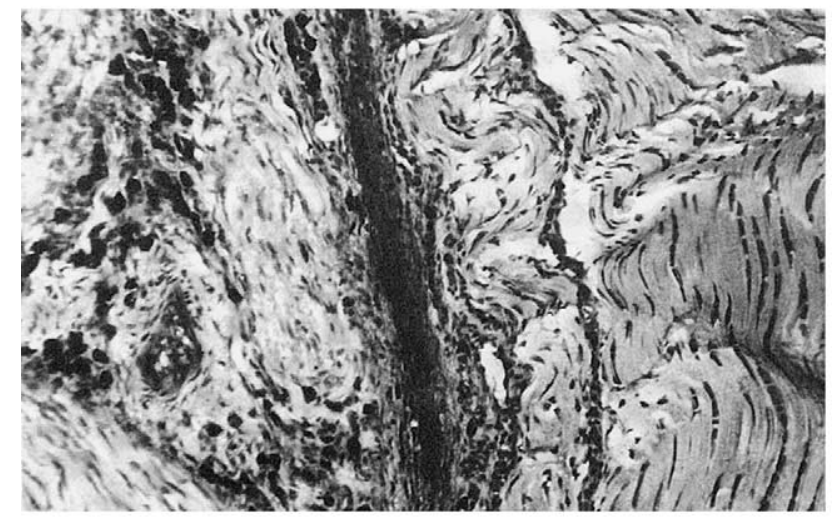

(C)

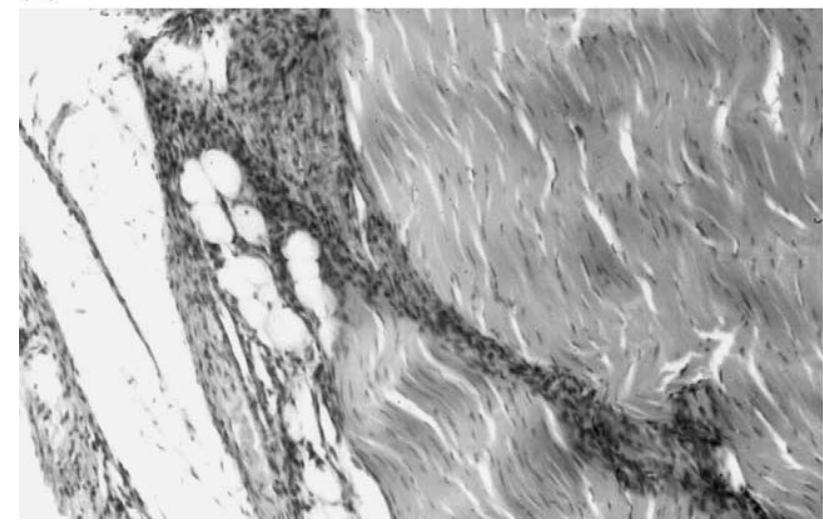

(B)

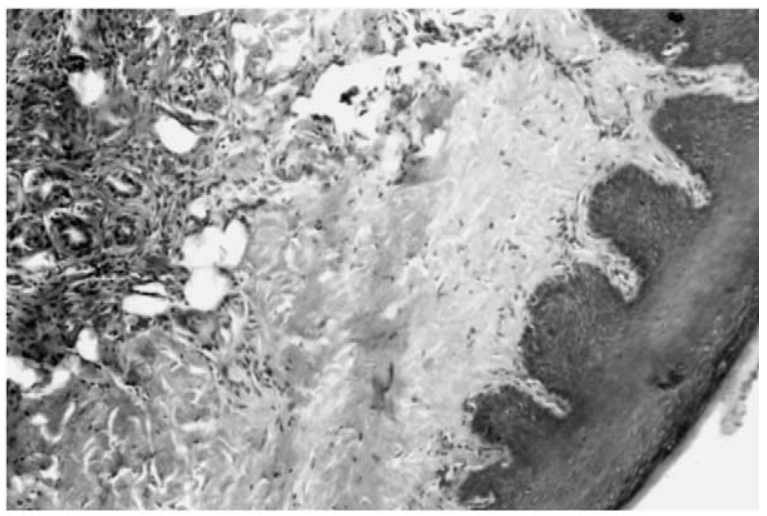

(D)

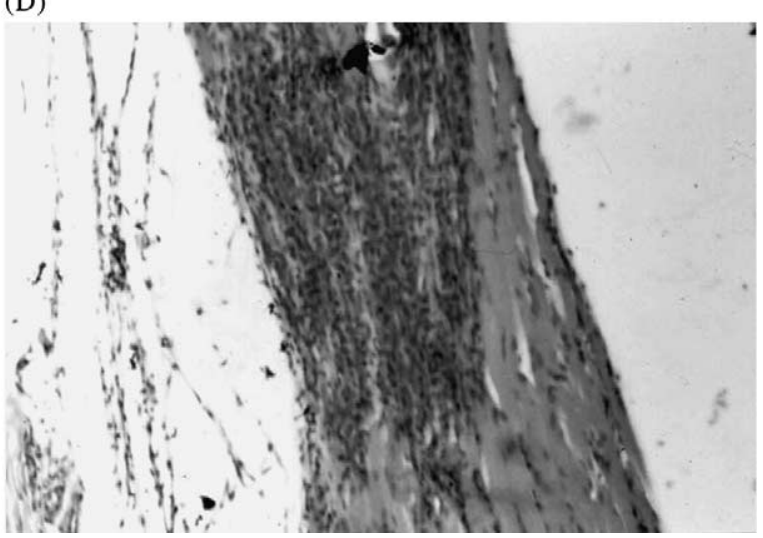

FIG. 3. Three days after tendon injury in the rat $(\times 10$, haematoxylin and eosin). (A) Control, no treatment: the subcutaneous tissue is edematous and is invaded by inflammatory cells that are mostly monocytes/macrophages and there are some darkly staining mast cells in close proximity to the tendon. Reproduced by kind permission from Martin Dunitz Ltd (1997). (B) LNAME treatment: edema in the subcutaneous tissue that is heavily infiltrated by larger mononuclear cells with larger nuclei than those in control injuries. Greater numbers of mast cells are also seen. (C) L-NAME treatment: severe adherence of large numbers of mononuclear cells to tendon. This is not normally seen in control injuries. (D) L-NAME treatment: extraordinarily severe adherence of large numbers of mononuclear cells to muscle. Not apparent in any of the control injured paws. 
(A)

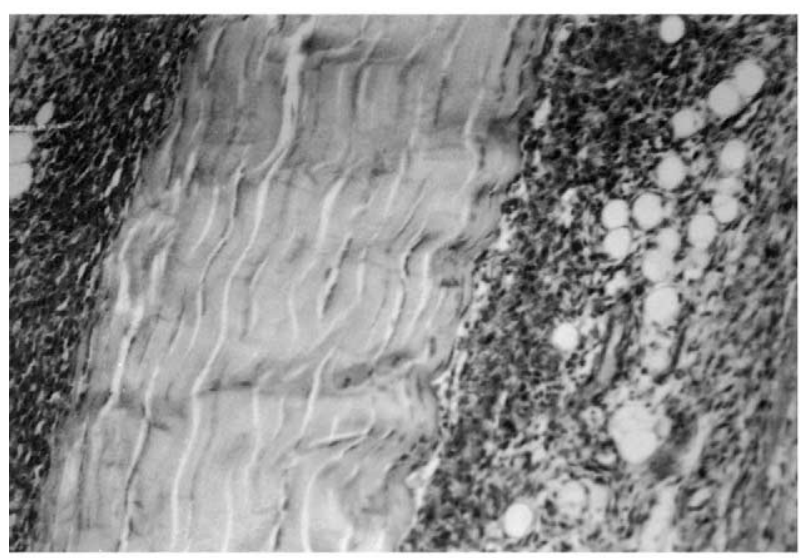

(B)

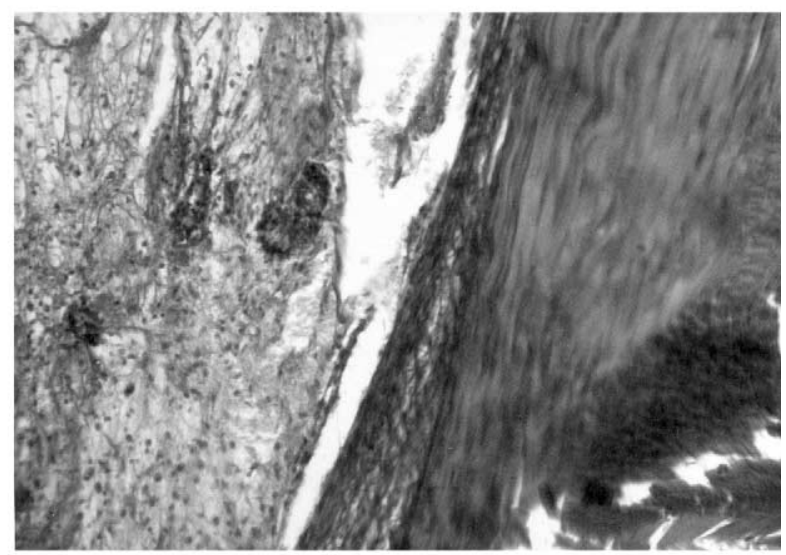

(C)

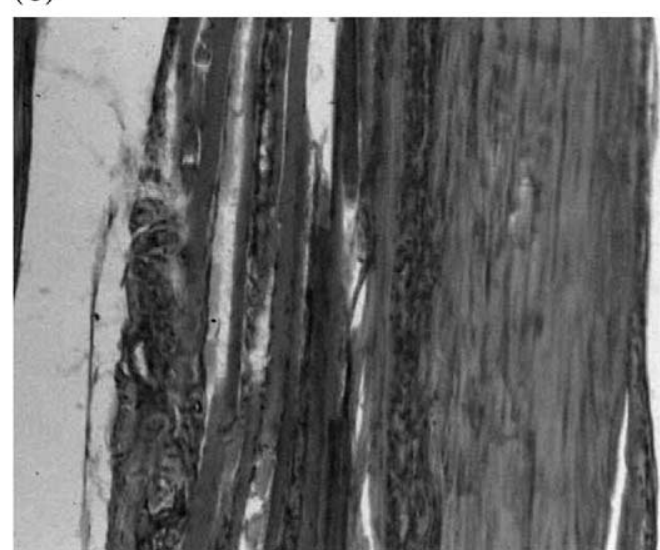

FIG. 4. Three weeks after tendon injury in the rat $(\times 10$, haematoxylin and eosin). (A) Control, no treatment: inflammatory cell numbers in the subcutaneous tissue is much reduced, although mononuclear cells are still present in the extensive areas where adhesions have formed. In these areas the synovial space is completely obliterated by adhesion formation. Reproduced by kind permission from Martin Dunitz Ltd (1997). (B) and (C) L-NAME treatment: inflammatory cell accumulation in the subcutaneous tissue is still severe and persistent, and large numbers of mononuclear cells are present in the areas where adhesions have formed between the synovium and tendon. The adhesions between tendon and synovium have increased and matured.

(A)

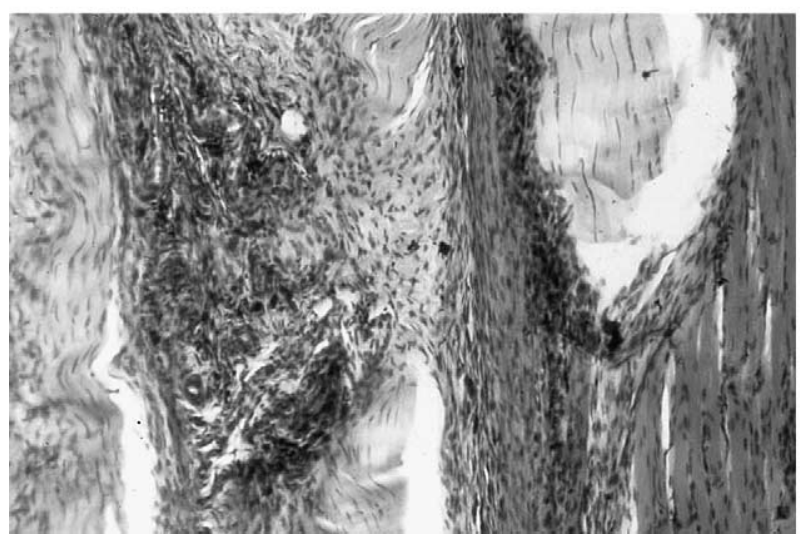

(B)

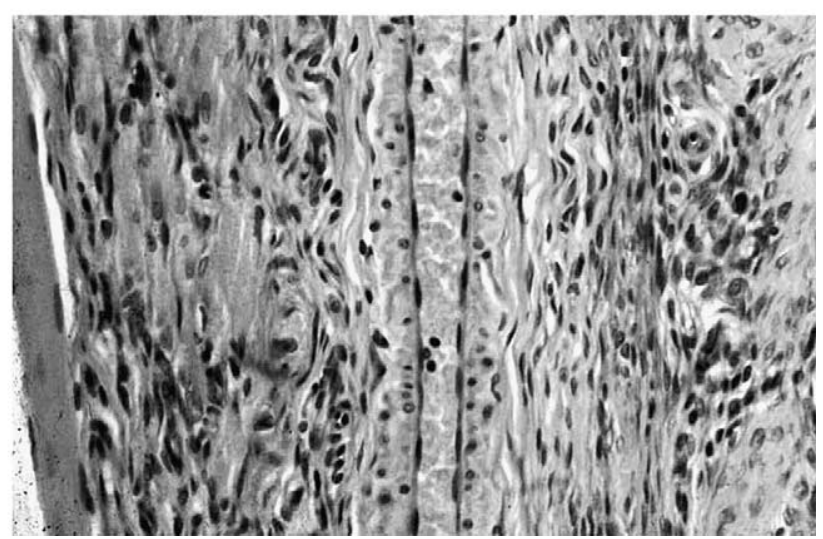

FIG. 5. Four weeks after tendon injury in the L-NAME-treated rat: $(A) \times 10$ and $(B) \times 40$, haematoxylin and eosin. Increased numbers of inflammatory cells in the subcutaneous tissues, in muscle and in tendon. Mononuclear cells are much larger (B) than at 2 or 3 weeks. There are few areas of clear synovial canal, and the tendon has completely merged with the interstitial tissues in some areas. 
(A)

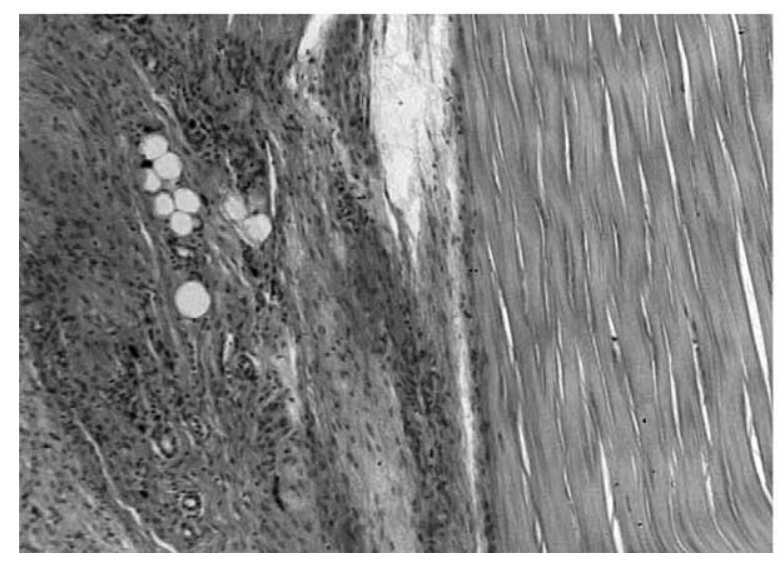

(B)

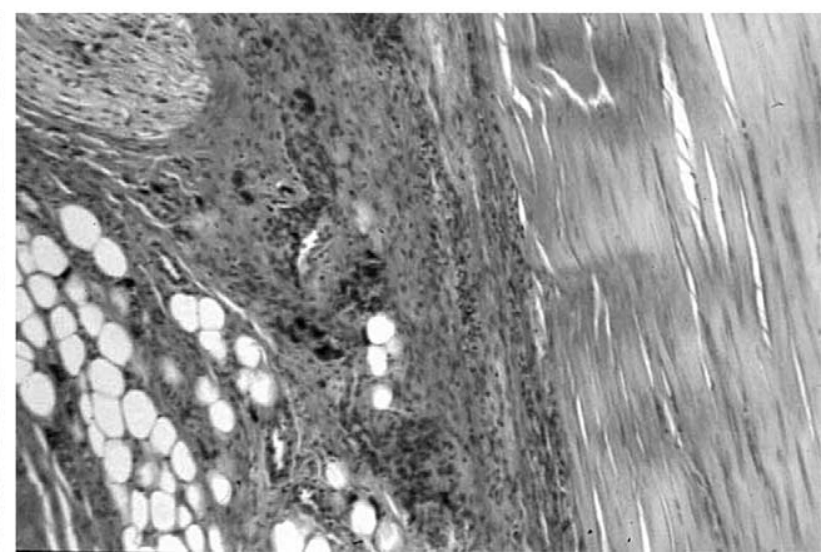

FIG. 6. Five weeks after tendon injury in the L-NAME-treated rat $(\times 10$, haematoxylin and eosin). Chronic inflammatory response still evident with a persistent accumulation of large macrophages and mast cells. Subcutaneous tissue is infiltrated with large numbers of inflammatory cells (A and B). Tendon is completely adherent to synovium (B).

\section{Two weeks}

In both groups, there was an overall reduction in the degree of inflammatory cell infiltration in the subcutaneous tissues and around the tendon, although the relative differences between the two groups were maintained. The mononuclear cell bodies and their nuclei were smaller and more rounded than those observed 3 days following injury and the differences were most marked in the paws of animals treated with L-NAME. In both groups the tendon had become adherent to synovium in most injured areas. The adhesions in the L-NAME-treated animals were more cellular than in control injuries.

\section{Three weeks}

In control injured paws the inflammatory cell infiltration was much reduced in the subcutaneous tissues, although mononuclear cells were still present in the extensive areas where adhesions had formed (Fig. $4 \mathrm{~A}$ ). In contrast, the paws of animals treated with LNAME showed a severe and persistent inflammatory cell accumulation (Fig. 4B,C). Most of these cells were of moderate size with relatively small nuclei. Fibrocellular adhesions between tendon and synovium had increased and matured.

\section{Four weeks}

While the inflammatory cell infiltrate in control injured paws continued to decrease, the paws of animals treated with L-NAME showed increased numbers of inflammatory cells in the subcutaneous tissues, in muscle and in tendon (Fig. 5A,B). These mononuclear cells were now much bigger than they were at 2 and 3 weeks. In many areas the histological appearances were similar to those observed 3 days following injury in L-NAME-treated paws, except for the absence of edema. Mast cells had once more begun to accumulate in larger numbers than at previous time intervals and often lay close to tendon. There were few areas of clear synovial canal and further merging of tendon with the interstitial tissues had occurred.

\section{Five weeks}

The inflammatory response in control injured paws had settled almost completely apart from the presence of some residual mononuclear cells in the fibrocellular adhesions. The appearances in the LNAME-treated animals were identical to those observed at 4 weeks (Fig. 6A,B). The treatment with LNAME had elicited a chronic inflammatory response characterised by the persistent accumulation of large macrophages and mast cells. Figure 7A,B confirm that the inflammatory cell population in histological sections of control 3-week-injured and L-NAME 4week-injured paws consist mainly of macrophage cells. The sections were labelled with the macrophage marker ED1.

\section{Discussion}

We first examined the effects of administration of a single dose of L-NAME, immediately after wounding, on subsequent NOS activity, measured by the release of nitrite into the medium, within the injured paw. We compared the release of nitrite from tendon/synovium explant cultures taken from control rats with uninjured paws and rats with crush-injured paws with and without L-NAME treatment. Our results indicate that nitrite production and hence NOS activity is doubled at the acute phase of tendon wound healing, and we can prevent this by administering a single dose of L-NAME immediately after injury. In a study of rat wound healing, Albina et al. ${ }^{14}$ 
showed that L-arginine (the substrate for the enzyme NOS) is important for efficient wound healing. They reported that arginine is metabolised by the NO pathway during the first 3 days of wound healing, and later on in the wound healing process arginine was metabolised by the arginase pathway. They concluded that the sequence of events can be explained by the fact that immediately after wounding, NO may provide vasodilatation and microbiostasis, which are both important events for efficient wound healing.

The importance of NO in wound healing is due to its many anti-inflammatory properties, including reduction of platelet aggregation, contribution to antimicrobial defense mechanisms as well as reducing proinflammatory cytokine production by endothelium. $^{9-11} \mathrm{NO}$ also inhibits the expression of Pselectin and two other endothelial cell adhesion molecules involved in adherence and transendothelial migration of monocytes and lymphocytes, namely intracellular adhesion molecule-1 (ICAM-1) and vascular cell adhesion molecule-1 (VCAM-1). ${ }^{15}$ When endogenous NO production was blocked by administration of L-NAME in the current study, there was extraordinarily severe adherence of large numbers of mononuclear cells to tendon and to muscle features that were not apparent in any of the control injured paws. Liu et al. ${ }^{16}$ observed that administration of L-NAME induced a significant augmentation of Pselectin and ICAM-1 gene expression and polymorphonuclear neutrophil (PMN) accumulation in a model of hepatic ischemia-reperfusion in rats. It was hypothesised that at sites of inflammation, the increase in NO production by endothelium, macrophages, and vascular smooth muscle cells is the essential compensatory response for maintaining organ blood flow and inhibiting PMN adhesion and platelet aggregation. P-selectin is important in pro- moting rolling and adherence of leukocytes to the endothelium. These effects on leukocyte rolling act to capture leukocytes and bring them into contact with the endothelium, where they can undergo firm adhesion, a process regulated by other adhesion molecules (i.e. ICAM-1 and VCAM-1). ${ }^{16}$ Thus, inhibition of NO production by L-NAME is likely to favour increased circulating leukocyte activation, adherence to endothelium and transendothelial migration.

It is difficult to explain why a single systemic dose of L-NAME administered at the time of injury produces such long-lasting effects. Perhaps it initiates a cascade of autocrine and paracrine events that are not reversed as they would be normally when wound healing mononuclear cells undergo apoptosis 2-3 weeks following injury. ${ }^{17}$ In this regard, it may be relevant that NO induces macrophage apoptosis and, in its absence, macrophages may continue to survive and thrive.

A parallel situation occurs in relation to systemic TGF- $\beta$ administration in animals with impaired wound healing potential. Beck et al. ${ }^{18}$ have shown that a single intravenous dose of TGF- $\beta$ administered $24 \mathrm{~h}$ prior to cutaneous wounding in diabetic or corticosteroid-treated rats promotes rapid wound healing, which does not otherwise take place. They have suggested that TGF- $\beta$ somehow primes circulating leukocytes to continue to express repair cytokines for a prolonged period throughout wound healing.

Increased interstitial accumulation of inflammatory cells and the development of fibrosis have been shown to occur in another experimental model of injury. Rats given 3 days of angiotensin II treatment that has been preceded by the chronic administration of L-NAME for 2 weeks develop interstitial cardiac fibrosis accompanied by chronic mononuclear cell infiltration. ${ }^{19}$ This is accompanied by increased
(A)

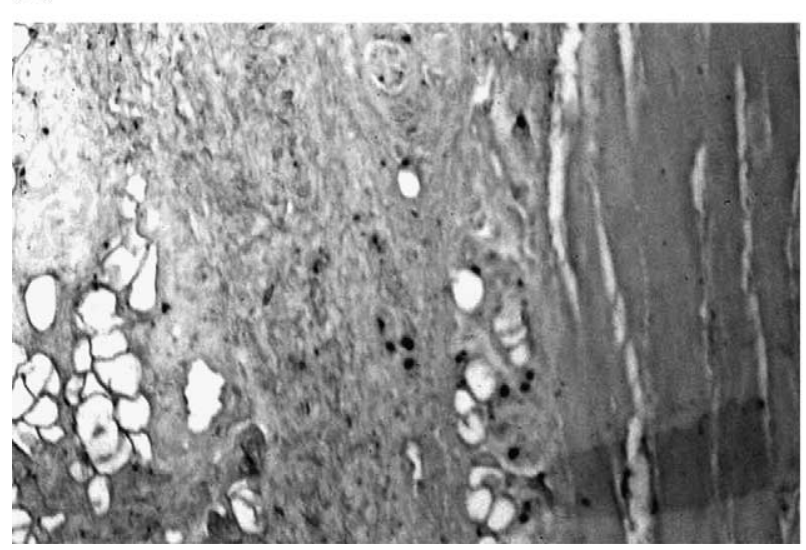

(B)

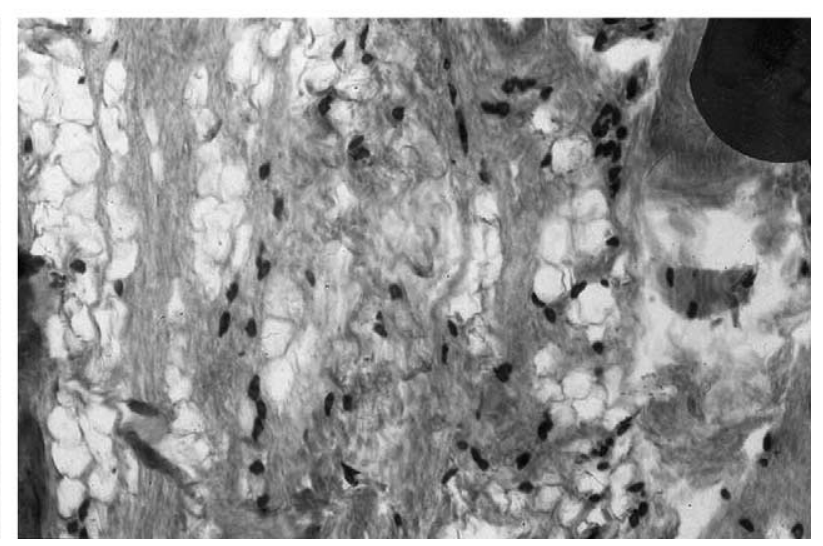

FIG. 7. (A) Three weeks after tendon injury in an untreated wound in the rat $(\times 10,5$-bromo-4-chloro-3 indolyl phosphate/nitroblue tetrazolium) showing small numbers of macrophages (labelled with the macrophage marker ED1) in the subcutaneous tissue. (B) Four weeks after tendon injury in L-NAME-treated rats $(\times 40,5$-bromo-4-chloro-3 indolyl phosphate/nitro-blue tetrazolium) showing increased numbers of macrophages (labelled with the macrophage marker ED1) in the subcutaneous tissue. Macrophages are larger than those in the control injury group. 
steady-state mRNA levels for matrix genes including a 60-fold increase in fibronectin mRNA. The administration of either angiotensin II or L-NAME alone in these animals was not sufficient to promote inflammatory cell accumulation or fibrosis, and the timing of L-NAME administration was crucial. ${ }^{19}$ A paradigm may be emerging whereby priming of tissues by injury may permit L-NAME to induce a chronic inflammatory state by autocrine and paracrine means. TGF- $\beta$ released in response to injury is likely to participate in this process as it also inhibits the production of NO. TGF- $\beta$ promotes adhesion formation in uninjured and injured peritoneum while inhibition of TGF- $\beta$ during the early inflammatory phase of wound healing greatly reduces adhesion formation around the tendon. The greatly increased levels of adhesion of leukocytes to tendon and muscle and the development of severe peritendinous adhesions in our study may be indicative of a chronic imbalance in the expression of TGF- $\beta$ (which is likely to be overexpressed) and NO (which is likely to be underexpressed).

Adhesion formation between the injured mesothelial tissue represents an inflammatory response to trauma and NO, an endogenous anti-adhesive molecule, may be important in the early stages of repair. So, if NO production is inhibited immediately after tendon crush injury, the mechanisms necessary for efficient repair may be compromised and thus an inflammatory reaction that is initially acute will fail to subside and a chronic inflammatory response may supervene. The inflammatory response itself damages host tissues, and indeed the magnitude of neutrophil influx into acute inflammatory lesions has been shown to correlate with the intensity of the inflammatory response and subsequent tissue injury. $^{20,21} \mathrm{NO}$ can in fact block PMN infiltration to protect tissues, ${ }^{22}$ and administration of L-NAME immediately after wounding may tamper with this beneficial effect. Thus, the ability of blocking PMN infiltration may be one of the mechanisms by which this endogenous inflammatory mediator may, under normal circumstances, protect some tissues from damage; and in the case of injured tendon and synovium, NO could act to protect the healing tissue from an uncontrolled inflammatory response.

Taken together, the importance of $\mathrm{NO}$ as a vasodilator and microbiostatic agent (both important for efficient wound healing) and its ability to block PMN infiltration, and thus protect the damaged tissue, may play a role in the efficient healing and repair of tendon crush injuries.

\section{References}

1. Chang J, Thunder R, Most D, Longaker MT, Lineaweaver WC. Studies in flexor tendon wound healing: neutralizing antibody to TGF- $\beta 1$ increases postoperative range of motion. Plast Reconstr Surg 2000; 105: 148-155.

2. Border WA, Noble NA. Transforming growth factor $\beta$ in tissue fibrosis. $N$ Engl J Med 1994; 331: 1286-1292.

3. Chang J, Most D, Stelnicki E, et al. Gene expression of transforming growth factor beta-1 in rabbit zone II flexor tendon wound healing: Evidence for dual mechanisms of repair. Plast Reconstr Surg 1997; 100: 937-944.

4. Miyajima A, Chen J, Lawrence C, et al. Antibody to transforming growth factor-beta ameliorates tubular apoptosis in unilateral ureteral obstruction. Kidney Int 2000; 58: 2301-2313.

5. Brahmatewari J, Serafini A, Serralta V, Mertz PM, Eaglstein WH. The effects of topical transforming growth factor-beta 2 and anti-transforming growth factor-beta2,3 on scarring in pigs. J Cutan Med Surg 2000; 4 : $126-131$.

6. Gilbert RS, Herschman HR. Transforming growth factor beta differentially modulates the inducible NOS gene in distinct cell types. Biochem Biophys Res Commun 1993; 195: 380-384.

7. Kunz D, Walker G, Pfeilschifter J. Transforming growth factor-beta 2 inhibits interleukin 1 beta-induced expression of inducible NOS in rat renal mesangial cells. Inflamm Res 1997; 46: 327-331.

8. Knowles RG, Moncada S. NO synthases in mammals. Biochem J 1994; 298: $249-258$.

9. Lirk P, Hoffmann G, Rieder J. Inducible nitric oxide synthase-time for reappraisal. Curr Drug Targets Inflamm Allergy 2002; 1: 89-108.

10. Shi HP, Most D, Efron DT, Tantry U, Fischel MH, Barbul A. The role of iNOS in wound healing. Surgery 2001; 130: 225-229.

11. Schwentker A, Billiar TR. NO and wound repair. Surg Clin North Am 2003; 83: $521-530$.

12. Ferrini MG, Vernet D, Magee TR, et al. Antifibrotic role of inducible nitric oxide synthase. Nitric Oxide 2002; 6: 283-294.

13. Lin JH, Wang MX, Wei A, Zhu W, Diwan AD, Murrell GA. Temporal expression of nitric oxide synthase isoforms in healing Achilles tendon. $J$ Orthop Res 2001; 19: 136-142.

14. Albina JE, Mills CD, Barbul A, et al. Arginine metabolism in wounds. Am J Physiol 1988; 17: E459-E467.

15. De Caterina R, Libby P, Peng H-B, et al. Nitric oxide decreases cytokine induced endothelial activation. NO selectively reduces endothelial expression of adhesion molecules and proinflammatory cytokines. $J$ Clin Invest 1995; 96: 60-68.

16. Liu P, Yin K, Nagele R, Wong PY-K. Inhibition of NOS attenuates peroxynitrite generation, but augments neutrophil infiltration in hepatic ischemia-reperfusion. J Pharmacol Exp Ther 1998; 284: 1139-1146.

17. Desmouliere A, Redard M, Darby I, Gabbiani G. Apoptosis mediates the decrease in cellularity during the transition between granulation tissue and scar. Am J Pathol 1995; 146: 56-66.

18. Beck LS, DeGuzman L, Lee WP, Xu Y, Siegel MW, Amento EP. One systemic administration of TGF- beta 1 reverses age- or glutocorticoidimpaired wound healing. J Clin Invest 1993; 92: 2841-2849.

19. Hou J, Kato H, Cohen RA, Chobanian AV, Brecher P. Angiotensin IIinduced cardiac fibrosis in the rat is increased by chronic inhibition of nitric oxide synthase. J Clin Invest 1995; 96: 2469-2477.

20. Warren JS, Ward PA, Johnson KJ. Oxygen radicals as mediators of inflammation. In: Henson PM, Murphy RC, eds. Handbook of Inflammation, Vol 6: Mediators of the Inflammatory Process. Amsterdam: Elsevier/Science Publishers, 1989.

21. Qasim FJ, Mathieson PW, Sendo F, Thiru S, Oliveira DB. Role of neutrophils in the pathogenesis of experimental vasculitis. Am J Pathol 1996; 149: 81-89.

22. Secco DD, Paron JA, de Oliveira SH, Ferreira SH, Silva JS, Cunha F, de O. Neutrophil migration in inflammation: nitric oxide inhibits rolling, adhesion and induces apoptosis. Nitric Oxide 2003; 9: 153-164.

\section{Received 22 February 2004 \\ Accepted 12 March 2004}




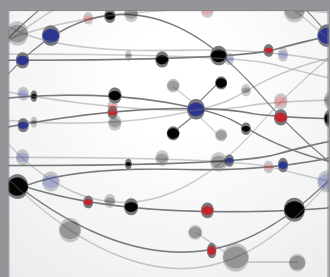

The Scientific World Journal
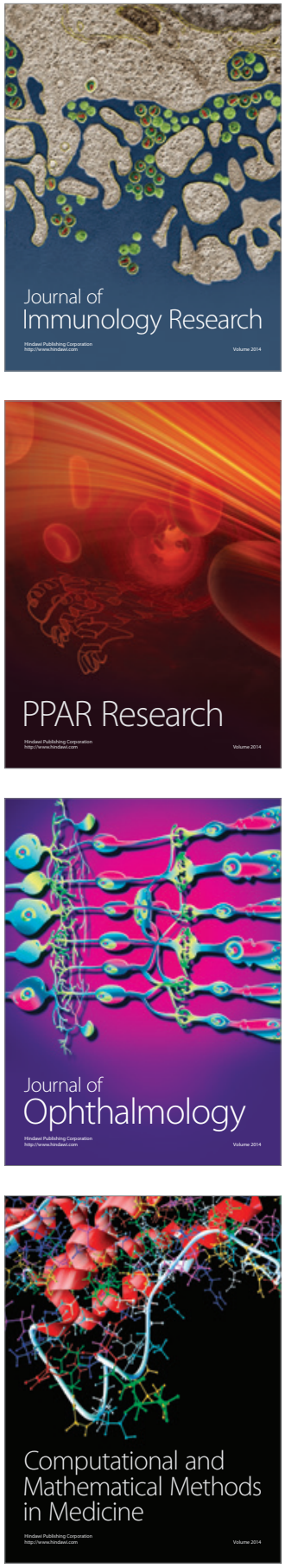

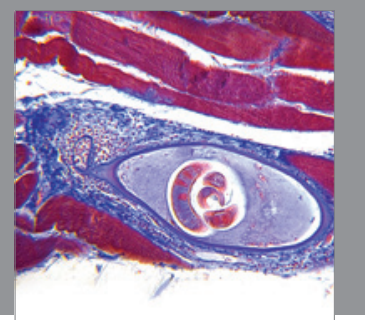

Gastroenterology

Research and Practice
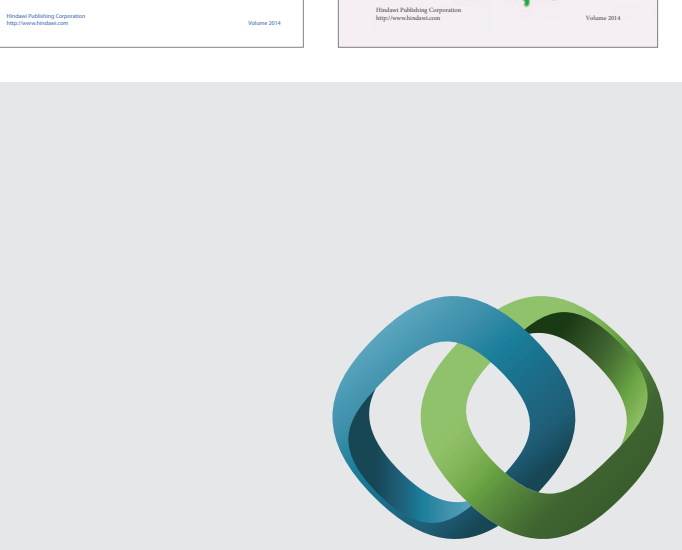

\section{Hindawi}

Submit your manuscripts at

http://www.hindawi.com
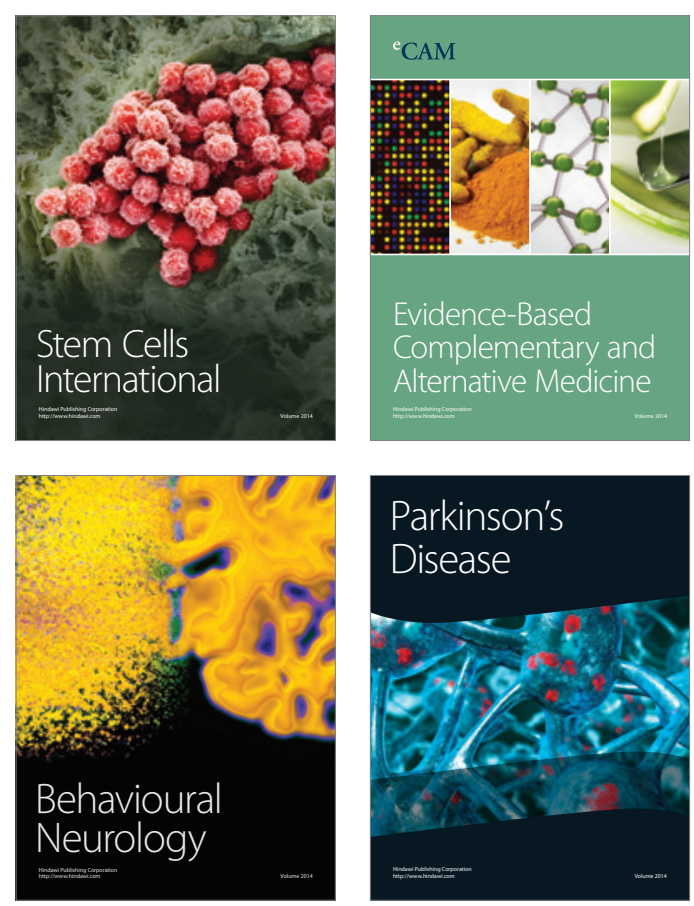

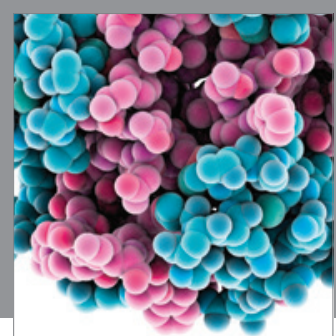

Journal of
Diabetes Research

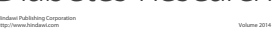

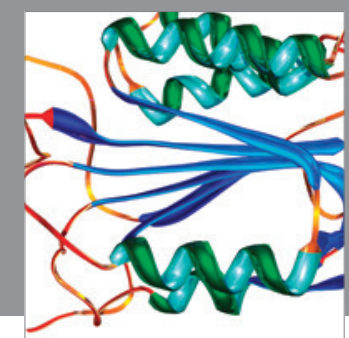

Disease Markers
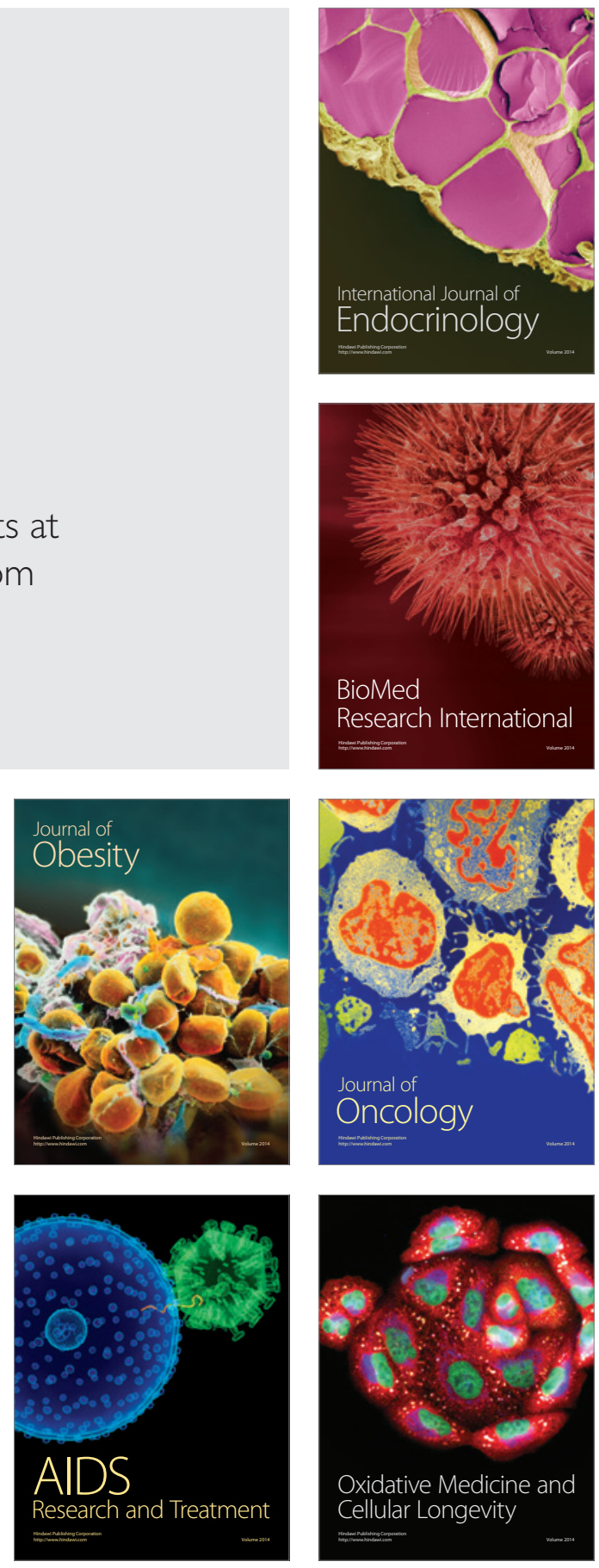